\title{
Distinct cytokine profiles of circulating mononuclear cells stimulated with Staphylococcus aureus enterotoxin A in vitro during early and late episodes of chronic osteomyelitis
}

\author{
Gabriella Freitas Ferreira ${ }^{1,2}$, Cícero Moraes ${ }^{1,3}$, \\ Alda Maria Soares da Silveira ${ }^{1,2}$, Rodrigo Correa-Oliveira ${ }^{4}$, \\ Andréa Teixeira-Carvalho ${ }^{4}$, Olindo Assis Martins-Filho ${ }^{4}$, Elizabeth Castro Moreno ${ }^{5}$, \\ Luiz Simeão do Carmo ${ }^{6}$, Lucia Alves de Oliveira Fraga ${ }^{1,2} /{ }^{+}$, Luiz Cosme Cotta Malaquiass, ${ }^{1,2,7}$
}

\footnotetext{
1Núcleo de Pesquisa em Imunologia ²Programa de Pós-Graduação em Ciências Biológicas, Universidade Vale do Rio Doce, Governador Valadares, MG, Brasil ${ }^{3}$ Hospital Municipal, Governador Valadares, MG, Brasil ${ }^{4}$ Instituto René Rachou-Fiocruz, Belo Horizonte, MG, Brasil ${ }^{5}$ Fundação Hemominas, Belo Horizonte, MG, Brasil ${ }^{6}$ Departamento de Parasitologia e Microbiologia, Instituto de Ciências Biológicas, Universidade Federal de Minas Gerais, Belo Horizonte, MG, Brasil `Laboratório de Microbiologia e Imunologia Básica, Departamento de Ciências Biomédicas, Universidade Federal de Alfenas, Alfenas, MG, Brasil
}

We investigated the cytokine profile of peripheral mononuclear cells from chronic osteomyelitis (OST) patients following in vitro stimulation with staphylococcal enterotoxin A (SEA). We demonstrate that stimulation with SEA induced prominent lymphocyte proliferation and high levels of tumour necrosis factor (TNF)- $\alpha$, interleukin (IL)-4 and IL-10 secretion in both OST and non-infected individuals (NI). Even though stimulation with SEA had no impact on IL-6 production in either patient group, the baseline level of IL-6 production by cells from OST patients was always significantly less than that produced by cells from NI. After classifying the osteomyelitic episodes based on the time after the last reactivation event as "early" (1-4 months) or "late" osteomyelitis (5-12 months), we found that increased levels of TNF- $\alpha$ and IL-4 in combination with decreased levels of IL-6 were observed in the early episodes. By contrast, increased levels of IL-10, IL-2 and IL-6 were hallmarks of late episodes. Our data demonstrate that early osteomyelitic episodes are accompanied by an increased frequency of "high producers" of TNF- $\alpha$ and $I L-4$, whereas late events are characterised by increased frequencies of "high producers" of IL-10, IL-6 and IL-2. These findings demonstrate the distinct cytokine profiles in chronic osteomyelitis, with a distinct regulation of IL-6 production during early and late episodes.

Key words: Staphylococcus aureus - enterotoxin A - chronic osteomyelitis - cytokines - IL-6

Osteomyelitis is a bone disease characterised by progressive inflammatory destruction of the infected bone and recruitment of osteocytes to the site of infection. In adults, osteomyelitis is usually a complication of open wounds involving the bone and typically results from fractures and/or surgery. The risk and severity of infection can be increased by the presence of a foreign body, such as metallic or prosthetic devices (Lew \& Waldvogel 2004). Between $0.4-7 \%$ of trauma and orthopaedic operations have been reported to be complicated by osteomyelitis (Haas \& McAndrew 1996, Lew \& Waldvogel 2004). Furthermore, this infection can also develop in non-injured bone after bacteraemia when the infection involves the axial skeleton; this type of infection is most often seen in prepubertal children and elderly patients (Roesgen et al. 1989, Haas \& McAndrew 1996).

Financial support: FAPEMIG, CNPq, CPqRR/FIOCRUZ-MG, UNIVALE

+ Corresponding author: luciaalvesfraga@yahoo.com.br

Received 23 June 2011

Accepted 1 February 2012
Up to $30 \%$ of osteomyelitic infections become chronic despite appropriate medical and surgical intervention, causing both economic losses and personal morbidity and mortality (Lew \& Waldvogel 2004). Most research has focused on improving the surgical and medical treatment of osteomyelitis, but little progress has been made towards understanding the underlying pathology.

Staphylococcus aureus is the microorganism most frequently isolated from posttraumatic and haematogenous osteomyelitis (Lew \& Waldvogel 1997, Jorge et al. 2010). The virulence of this bacterium is associated with its ability to penetrate the bone tissue, evade the host defences and secrete exotoxins, such as the superantigen staphylococcal enterotoxin A (SEA) (Nair et al. 2000), which $57 \%$ of $S$. aureus strains isolated from chronic osteomyelitis patients (OST) are able to produce (Sourek et al. 1979). Elevated SEA production has also been reported to be associated with other diseases, such as atopic dermatitis (Naffa et al. 2006, Adwan et al. 2006, Cho et al. 2009).

The causes of osteomyelitis are multifactorial and are mainly influenced by local factors related to the bone lesion and the microorganisms present in the bone. However, inherited factors and dysfunctions in cellular immunity may also be relevant (Yoon et al. 1999, Tsezou et al. 2008). 
Schluter et al. (1991) demonstrated that patients suffering from chronic osteomyelitis exhibited diminished responses in cutaneous hypersensitivity tests and reduced phytohaemagglutinin-induced $\mathrm{T}$ lymphocyte proliferation when compared with healthy individuals. These patients presented with normal serum IgG levels and normal $S$. aureus lysate-induced B lymphocyte proliferation, but a reduced $\mathrm{CD}^{+} / \mathrm{CD}^{+} \mathrm{T}$ cell ratio. Pudil et al. (1993) also observed a significant reduction in CD4 ${ }^{+}$ $\mathrm{T}$ cells in chronic OST patients compared with non-infected individuals (NI).

Other alterations in the immune system have been reported in chronic OST patients, including reduced complement activity, a reduced phagocytic and microbicidal potential of macrophages and granulocytes and reduced $\mathrm{C} 3 \mathrm{~b}$ receptor expression in phagocytes (Hierholzer \& Hierholzer 1982, Schluter et al. 1991, Pudil et al. 1993). Furthermore, nitric oxide (NO) production by phagocytes has been shown to diminish the mortality of rats experimentally infected with $S$. aureus (Sasaki et al. 1998).

Increased expression of pro-inflammatory cytokines, such as interleukin (IL)-1, IL-6 and tumour necrosis factor (TNF)- $\alpha$, has been reported in OST patients (Klosterhalfen et al. 1996, Evans et al. 1998). Specifically, cytokines such as IL-6, TNF- $\alpha$, and IL-4 are directly involved in the destruction of bone and the regulation of osteoclast activity in OST patients and, therefore, may play a role in disease pathogenesis (Pesanti \& Lorenzo 1998, Fullilove et al. 2000, Balto et al. 2001, Marriott et al. 2004).

Because the cellular immune response during chronic staphylococcal osteomyelitis has not been fully characterised in humans, we investigated the contribution of antigen-induced cellular reactivity, cytokine production in peripheral blood mononuclear cells (PBMCs) and serum NO levels to disease pathogenesis in chronic staphylococcal OST patients.

\section{SUBJECTS, MATERIALS AND METHODS}

Study population - Twenty individuals with chronic staphylococcal osteomyelitis were selected from patients attending the Municipal Hospital in Governador Valadares, state of Minas Gerais (MG), Brazil, over a period of eight months. The inclusion criteria were the presence of $S$. aureus at the site of infection, the presence of fistulas over a less-than-one-year period and bone sequestra (i.e., areas of dead bone tissue) as indicators of chronic osteomyelitis. The chronic OST group comprised 14 males and six females, with an age range of 10-60 years (mean, 35 years). In most cases (18/20), the bone infection was caused by trauma and the most commonly affected bones were the tibia and femur. The mean hospitalisation time was 25 days and the average time since the last acute recurrence was 5.2 months, as assessed by clinical manifestations (i.e., fever, intense bone pain, presence of fistulas and purulent secretions). The patients were further sub-grouped based on the time since the last acute recurrence. Considering that chronic osteomyelitis is defined by some authors as when the infection has been present for more than one month (Jevon et al. 1999, Brady et al. 2008, Howell \& Goulston 2011) and by others as an infection lasting longer than six months (Gillespie \&
Allardyce 1990, Bansal et al. 1992), we established two groups of chronic OST patients: early-stage patients (i.e., those in which 1-4 months had elapsed since the most recent acute recurrence) and late-stage patients (i.e., those in which 5-12 months had passed since the last relapse). The median recurrence time in the individuals was four months and both the early stage and late stage groups were composed of 10 OST patients.

The control group comprised 20 healthy volunteers from the University Vale do Rio Doce (14 men and 6 women), with an average age of 35 years. They were not suffering from any chronic infection or illness and did not receive any previous treatment.

The project was submitted to and approved by the Ethical Committees of University Vale do Rio Doce (protocol 04/2003). Written consent was obtained from all participants in the study.

Cell proliferation assay and in vitro cytokine production - For each assay, $30 \mathrm{~mL}$ of peripheral blood from one or more OST patients and one or two healthy donors were simultaneously collected in heparinised Vacutainer tubes. Plasma was separated from whole blood via centrifugation and stored at $-20^{\circ} \mathrm{C}$ until analysis. PBMCs were isolated from the blood samples using Ficoll-Hypaque density centrifugation, as described by Gazzinelli et al. (1983). Over $95 \%$ of the cells isolated from the gradient interface were viable, as detected using Trypan blue exclusion. PBMCs were cultured in RPMI-1640 medium (Sigma, St. Louis, MO, USA) supplemented with 1.6\% Lglutamine $(200 \mathrm{mM}), 3 \%$ antibiotic/antimycotic mixture $(10,000 \mathrm{U} / \mathrm{mL}$ penicillin, $10 \mathrm{mg} / \mathrm{mL}$ streptomycin and $25 \mu \mathrm{g} / \mathrm{mL}$ amphotericin B, Sigma) and 5\% inactivated normal human serum (Sigma). For in vitro cytokine production, the cells were cultured in duplicate in 24-well, flat-bottom plates, each well contained $800 \mu \mathrm{L}$ culture medium, $100 \mu \mathrm{L}$ PBMCs $\left(1 \times 10^{7}\right.$ cells $\left./ \mathrm{mL}\right)$ resuspended in complete RPMI-1640 medium and $100 \mu \mathrm{L}$ SEA (1.0 $\mu \mathrm{g} / \mathrm{mL}$ ). Purified enterotoxin A was obtained from the Staphylococcus Laboratory (Fundação Ezequiel Dias, Belo Horizonte, MG, Brazil) and prepared as described by Cardoso et al. (1999). A previously established doseresponse curve was used to determine the optimum antigen and cell concentrations for the assay. In the unstimulated control cultures, $100 \mu \mathrm{L}$ PBMC suspension $\left(1 \times 10^{7}\right.$ cells $/ \mathrm{mL}$ ) was incubated with $100 \mu \mathrm{L}$ culture medium instead of antigen. The cell cultures were maintained for three days at $37^{\circ} \mathrm{C}$ in $5 \% \mathrm{CO}_{2}$ in a humidified atmosphere. Following incubation, the cell-free culture supernatants were collected and stored at $-20^{\circ} \mathrm{C}$ until analysis. For the proliferation assays, $25 \mu \mathrm{L}$ PBMCs $\left(1 \times 10^{7}\right.$ cells/ $\mathrm{mL}$ ) in complete RPMI-1640 medium was mixed with $150 \mu \mathrm{L}$ culture medium and $25 \mu \mathrm{L}$ SEA $(1.0 \mu \mathrm{g} / \mathrm{mL})$. All cultures were carried out in triplicate in 96-well plates and maintained for three days at $37^{\circ} \mathrm{C}$ in $5 \% \mathrm{CO}_{2}$ and a humidified atmosphere. During the last $6 \mathrm{~h}$ of culture, $\left[{ }^{3} \mathrm{H}\right]$-thymidine $(0.5 \mu \mathrm{Ci} /$ well, Amersham, UK) was added to each well and after $6 \mathrm{~h}$ had elapsed, the cells were harvested and thymidine incorporation was measured in a liquid scintillation counter (Beckman, Irvine, CA, USA). The lymphocyte proliferation data are presented as mean counts per minute for each group. 
Cytokine quantitation - A Cytometric Bead Array (CBA) Kit (Becton Dickinson Biosciences Pharmingen, San Diego, CA) was used for quantitative analysis of the cytokine levels. The following cytokines were evaluated, as recommended by the manufacturer: interferon (IFN)- $\gamma$, TNF- $\alpha$, IL-6, IL-4, IL-10 and IL-2. Briefly, 25 $\mu \mathrm{L}$ of culture supernatant or dilutions of the standards was added to $15 \mu \mathrm{L}$ of pooled beads and incubated. After incubation, the samples and standards were washed and centrifuged and $20 \mu \mathrm{L}$ of detection cocktail, consisting of six PE-conjugated $\mathrm{mAbs}$, was added to each tube, which were then incubated. Following incubation, the samples and standards were washed again and analysed using a FACSCalibur flow cytometer (Becton Dickinson). A total of 1,800 events/gate were acquired. A cytokine standard calibrator mixture was used for each assay to extrapolate the cytokine concentrations in the samples and data analysis was performed using BD Bioscience CBA software.

Serum NO levels - Serum samples from chronic OST and NI controls were tested using nitrate reductase. The enzyme was added to the serum samples and the nitrite content was determined via the Griess reaction, as described previously (Moshage \& Jansen 1998). The nitrite concentrations were determined at $540 \mathrm{~nm}$ using an automated ELISA plate reader and the results are expressed in $\mu \mathrm{M}$.

Statistical analyses - Statistical analyses were performed using the GraphPad Prism software package (San Diego, CA, USA). Kruskal-Wallis variance analyses with a post hoc Dunne's test were used to compare the cytokine profiles of the NI individuals and the patients from the early-stage or late-stage chronic OST groups. Significant differences $(p<0.05)$ among clinical groups are indicated by connected lines. The analyses comparing the cultures in the absence or presence of SEA were performed using the Mann-Whitney U-test and significant differences $(p<0.05$ or $p<0.005)$ are indicated. The frequency of individuals classified as low and highcytokine producers was compiled by establishing the global mean of each cytokine by taking all values from NI and OST patients, including data from non-stimulated and SEA-stimulated in vitro cultures (Fig. 1A) and classifying each individual as a low or high-cytokine producer, according to the established cut-off level for each cytokine (Fig. 1B).

After stimulation with SEA, a change in the frequency of high producers, either by one $\left(^{*}\right)$ or by two quartiles $(* *)$, is indicated in the figures with asterisks.

\section{RESULTS}

PBMCs from OST patients secrete higher levels of TNF- $\alpha, I L-4$ and IL-10 following in vitro stimulation with $S$. aureus enterotoxin $A$ - PBMC from OST patients and NI responded strongly to stimulation with SEA, demonstrating increased proliferation (Fig. 2). Moreover, in vitro stimulation with SEA induced increased secretion of TNF- $\alpha$, IL-4 and IL-10 by PBMCs from NI and OST individuals when compared with unstimulated control cultures. Although there was no difference in
IL-6 production between the unstimulated and stimulated PBMCs, the IL-6 level in the supernatants of PBMCs from OST patients was always significantly less than the level in the supernatants of PBMCs from NI. Additionally, the serum NO levels were significant lower in the OST patients than the levels in the NI (Fig. 2).

Distinct cytokine profiles during early and late osteomyelitic episodes - To evaluate potential differences between early (1-4 months) and late osteomyelitic episodes (5-12 months) we characterised the cytokine profile of PBMCs after in vitro incubation in either the absence or presence of SEA. Elevated levels of TNF- $\alpha$ and IL-4 and decreased levels of IL-6 were observed during early osteomyelitic episodes, whereas increased levels of IL-10, IL-2 and IL-6 were indicators of late osteomyelitic episodes (Fig. 3).

Establishing the cut-off for low and high cytokine production - We established the concept of high and low cytokine production to further understand the cytokine network in chronic OST patients. To do so, the global mean for each cytokine was calculated by taking the entire range of values obtained for all NI and infected patients. The global mean level for each cytokine was used to calculate the cut-offs and segregate all patients into either the high or low-cytokine production groups (Fig. 1A).

Increased frequency of high IFN- $\gamma, I L-10, I L-6$ and IL-2 producers in the late-stage chronic OST group - To characterise time-dependent and oscillating changes in the cytokine profile of PBMCs from chronic OST patients, the frequency of high-cytokine producers was evaluated in the presence or absence of SEA. SEA-stimulated PBMCs from late-stage OST patients exhibited increased production of IFN- $\gamma$, IL-10, IL- 6 and IL-2 and were considered "high"-cytokine producers. By contrast, early-stage OST patients demonstrated increased production of TNF- $\alpha$ and IL-4 in response to SEA (Fig. 1B).

Distinct cytokine signatures observed during early and late clinical episodes - A schematic representation highlighting the immunological response patterns observed in NI individuals and OST patients is displayed in Fig. 4. Compared with healthy individuals, the cytokine profile during chronic osteomyelitis is characterised by an increased frequency of high producers of TNF- $\alpha$ and IL-10. However, when the group of OST patients was divided into the early and late-stage groups, different response patterns emerged. We detected an increased frequency of high producers of IFN- $\gamma$, IL-10, IL- 6 and IL-2 in late-stage OST patients, whereas the early-stage OST group presented with a decreased frequency of high IL6 -producers and an increased frequency of high TNF- $\alpha$ and IL-4 producers.

\section{DISCUSSION}

The manner in which $S$. aureus interacts with the host tissues may facilitate invasion and enable it to evade the host immune response and release toxins throughout the infection process (Lew \& Waldvogel 2004, Howell $\&$ Goulston 2011). In patients with osteomyelitis, various cellular and humoral immunological deficits have been demonstrated (Gillespie \& Allardyce 1990, Schluter et 
al. 1991, Bansal et al. 1992, Sistermann et al. 1992, Wagner et al. 2003). In addition, experiments by Peters et al. (1995) demonstrated important alterations in tissuederived macrophages, resulting in a significant increase in anti-inflammatory macrophages.

There are no clear criteria that distinguish acute osteomyelitis from chronic osteomyelitis. In general, the patient is considered cured when he or she does not relapse over a year of follow-up (Dormans \& Drummond 1994, Carek et al. 2001, Asensi et al. 2004, 2007).

Today, standard diagnostic procedures to determine inflammatory activity include leukocyte counts, $\mathrm{C}$-reac- tive protein (CRP) levels, fibrinogen levels and the blood sedimentation rate. However, these parameters are nonspecific and can increase after a latent phase of days or even weeks (Harle 1989, Lew \& Waldvogel 2004, Howell \& Goulston 2011). Other parameters to estimate disease activity include measuring inflammatory mediators, such as the polymorphonuclear neutrophil (PMN) elastase level or neopterin level in blood and plasma samples (Peters et al. 1991). To improve upon the current available diagnostic tests, it is important to understand the role and dynamics of cytokines during early and late osteomyelitic episodes.

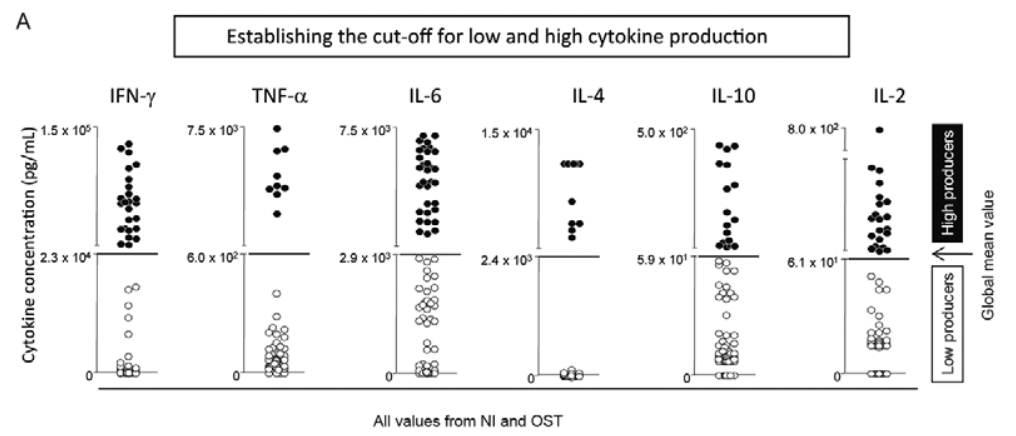

B

Frequency of individuals classified as low and high cytokine producers
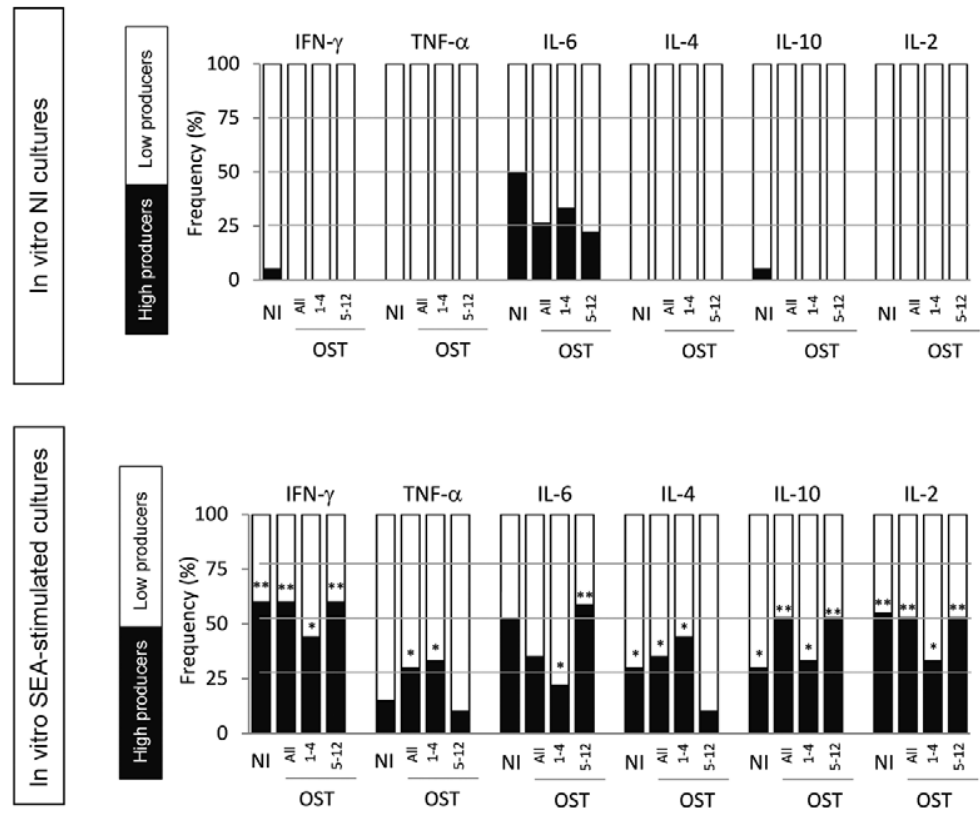

Fig. 1A: establishment of cut-off to segregate low (०) and high cytokine production (•) and frequency of individuals classified as high or low cytokine producers evaluated in the presence or in the absence of exogenous stimulus [staphylococcal enterotoxin A (SEA)]. A: scatter plot distribution highlight the global mean values for each cytokine $(\leftarrow)$ used as the edge (---) to define low and high producers. The global mean value was calculated using all data sets from non-infected (NI) and osteomyelitis (OST) groups and included the results from non-stimulated and SEA-stimulated in vitro cultures; B: the bars represent the frequency of high (घ) and low ( $\square$ ) producers for cytokine interferon (IFN)- $\gamma$, tumour necrosis factor (TNF)- $\alpha$, interleukin (IL)-6, IL-4, IL-10 and IL-2; *: the frequency of high producers moved one quartile after stimulation by SEA; **: the change of two quartiles. 
Among others factors, osteomyelitic relapses can result from the persistence of a foreign body, incomplete surgical debridement of bone sequestra or the duration and dose of the prescribed antibiotic (Haas \& McAndrew 1996, Carek et al. 2001, Lew \& Waldvogel 2004). In our study, all patients were treated and followed by the same physician and the majority took the same antibiotic (oxacillin). Patients with orthopaedic prostheses did not participate in the study. All results were based on the time since the last osteomyelitic relapse, which allowed us to establish stratified groups of chronic patients. Chronic OST patients were divided in early relapsers, who had a new osteomyelitic episode 1-4 months after the previous one, and late relapsers, who had a new osteomyelitic episode 5-12 months after the previous one, with four months representing the median interval of recurrence in the patients followed for this study.

Our data showed that serum NO levels were significantly lower $(p=0.04)$ in the OST group than in the healthy controls. In contrast, Asensi et al. (2007) found higher serum NO levels in OST patients homozygous for the NOS3 (27-bp repeat, intron 4) polymorphism than in healthy controls. However, when these authors compared the serum NO concentration in 65 OST patients carrying all NOS3 genotypes with healthy controls, they did not find any significant differences. Their cohort included both acute and chronic OST patients who were infected with different pathogens. In our study, we excluded acute OST patients and included only OST patients with $S$. aureus infection, which could explain the discordant results. Additionally, patients who are homozygous for the NOS3 polymorphism account for only a small percentage of OST patients (Asensi et al. 2007).

We detected significantly lower levels of IL-6 in the culture supernatants of PBMCs isolated from Staphylococcus-infected individuals than the PBMCs isolated from NI, both with and without antigenic stimulation (Fig. 2). In contrast to our results, Asensi et al. (2004) found increased plasma levels of IL-6 in OST patients. Again, they examined both acute and chronic patients, which could have contributed to the increased plasma levels of pro-inflammatory cytokines (e.g., IL-6, IL$1 \alpha, \mathrm{TNF}-\alpha)$ and CRP that they observed. Because we exclusively worked with chronic OST patients, the different phase of infection and the measurement of IL-6 in the supernatant instead of the serum might have contributed to the different concentrations reported in our study and previous ones.

When we regrouped individuals based on their relapse time, the characteristics of the IL- 6 response changed. After stimulation with SEA, the IL-6 level was significantly lower in the culture supernatants of PBMCs
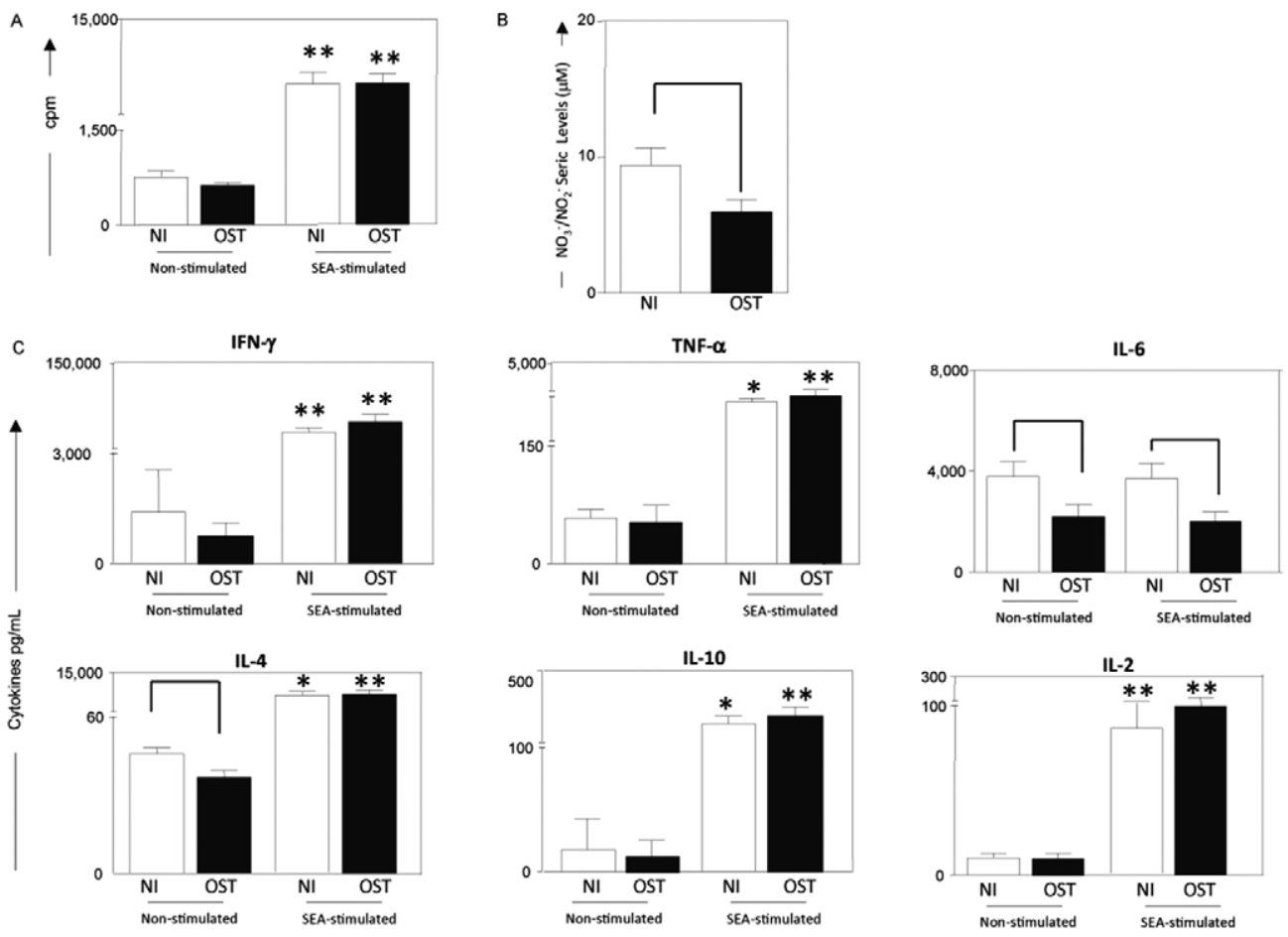

Fig. 2: proliferation of peripheral blood mononuclear cells (PBMC) (A), nitric oxide (NO) in serum (B) and cytokines levels (C) from patients with chronic staphylococcal osteomyelitis (OST) ( $\bullet$ ) and non-infected individuals (NI) (口). PBMC were stimulated with the culture medium only (non-stimulated cultures) or with staphylococcal enterotoxin A (SEA)-stimulated cultures. Results are expressed in counts per minute (cpm) (A). Seric levels of NO (nitrite + nitrate) are expressed in $\mu \mathrm{M}$ (B). Production of cytokines interferon (IFN)- $\gamma$, tumour necrosis factor (TNF)- $\alpha$, interleukin (IL)-6, IL-4, IL-10 and IL-2 are expressed in $\mathrm{pg} / \mathrm{mL}$ (C). Data represent mean results \pm standard errors. Within groups, significant differences between stimulated and non-stimulated cultures are identified by asterisks $(*: \mathrm{p}<0.05 ; * *$ : $p<0.005)$. Significant differences between groups are indicated by connecting lines $(\mathrm{p}<0.05)$. 
from early OST individuals than the level from NI individuals. In addition, when the IL-6 levels were compared within the same group, PBMCs from early OST patients produced significantly less IL-6 following stimulation with SEA when compared with the unstimulated control cultures. By contrast, PBMCs isolated from late OST patients demonstrated increased IL-6 following stimulation with SEA. These results reinforce the impact of on-going chronic disease, together with permanent antigenic stimulation of the immune system, which might contribute to the differences in IL-6 secretion between early and late episodes of chronic osteomyelitis.

During a chronic inflammatory process, IL-6 has a detrimental role that favours mononuclear cell accumulation at the site of injury. IL-6 plays a rather unexpected role in leukocyte recruitment in vivo. When complexed, IL-6 and IL-6R $\alpha$ can induce endothelial cells to secrete IL-8 and monocyte chemoattractant protein (MCP)-1 and express adhesion molecules. The IL-6/ IL-6R $\alpha$ complex is directly involved in the transition of inflammatory sites from a neutrophilic to monocytic pattern (Romano et al. 1997, Hurst et al. 2001, Marin et al. 2001).

Neutrophils stimulated with inflammatory cytokines for several hours will selectively produce MCP-1, but not IL-8. A transition from neutrophil to monocyte accumulation at the site of inflammation suggests that there is a progression of events that leads to both monocyte recruitment and apoptotic clearance of neutrophils. These events may increase the serum IL-6 level and provide the basis for the amplification step of the chronic inflammatory process (Jones et al. 2005). Therefore, in our system of chronic osteomyelitis, the source of the increased IL-6 in the late stage of infection (5-12 months) might be activated monocytes. In an experimental model, SEA was shown to bind to both major histocompatibility complex (MHC) class I and class II molecules, inducing IL- 6 and TNF- $\alpha$ and activating murine peritoneal macrophages. Therefore, both MHC class I and II may contribute to an in vivo response to staphylococcal superantigens (Abrahmsén et al. 1995, Beharka et al. 1995). However, this must be addressed in more detail in future experiments.

As proposed by some authors (Kaplanski et al. 2003, Gabay 2006), the main event causing the switch from acute to chronic inflammation is the recruitment of monocytes to the inflamed area and IL- 6 plays an important role in this transition. Furthermore, the increased production of IL-10 in stimulated PBMCs from late OST patients suggests that macrophages might par-
A

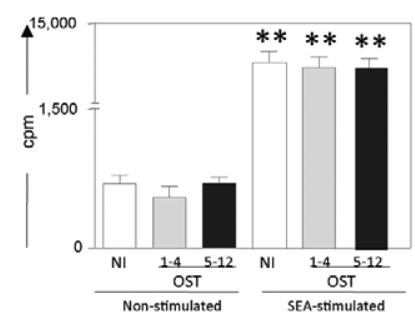

c
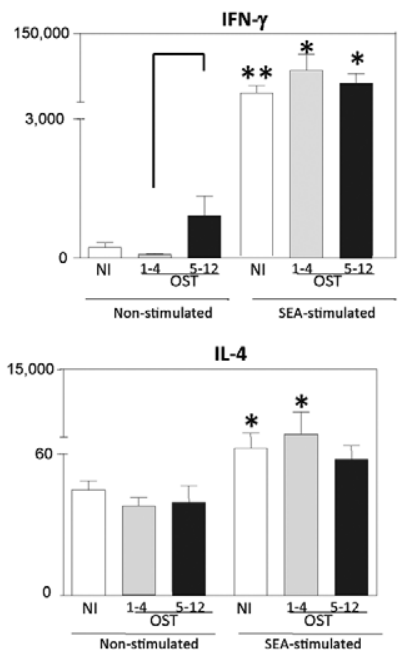

B
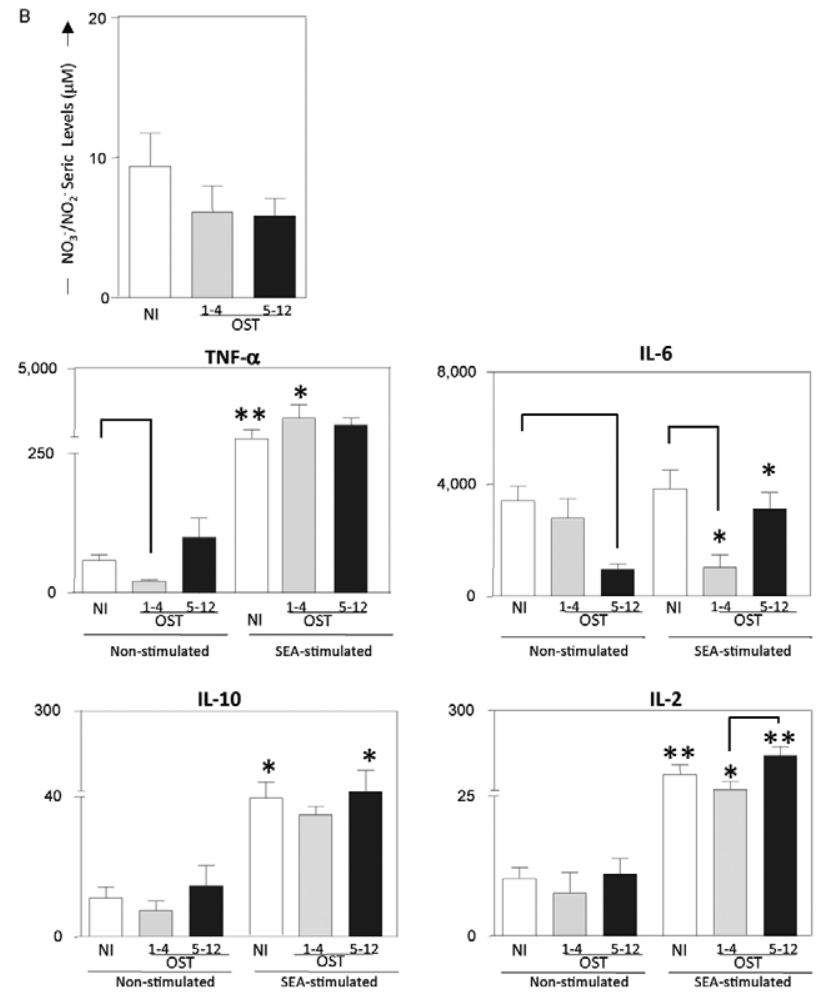

Fig. 3: proliferation of peripheral blood mononuclear cells (PBMC) (A), nitric oxide (NO) in serum (B) and cytokines levels (C) from patients

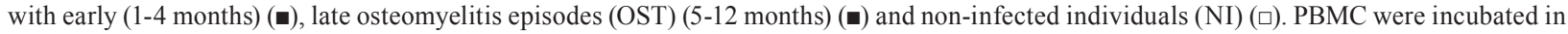
culture medium only (non-stimulated cultures) or with staphylococcal enterotoxin A (SEA)-stimulated cultures. Results are expressed in counts per minute (cpm) (A). Seric levels of NO (nitrite + nitrate $\mu \mathrm{M}$ ) are expressed in $\mu \mathrm{M}$ (B). Production of cytokines interferon (IFN)- $\gamma$, tumour necrosis factor (TNF)- $\alpha$, interleukin (IL)-6, IL-4, IL-10 and IL-2 are expressed in pg/mL (C). Data represent mean results \pm standard errors. Within groups, significant differences between stimulated and non-stimulated cultures are identified by asterisks $(*: \mathrm{p}<0.05 ; * *$ : $<0.005)$. Significant differences between groups are indicated by connecting lines $(\mathrm{p}<0.05)$. 
Overall cytokine signature triggered upon in vitro SEA-stimulation
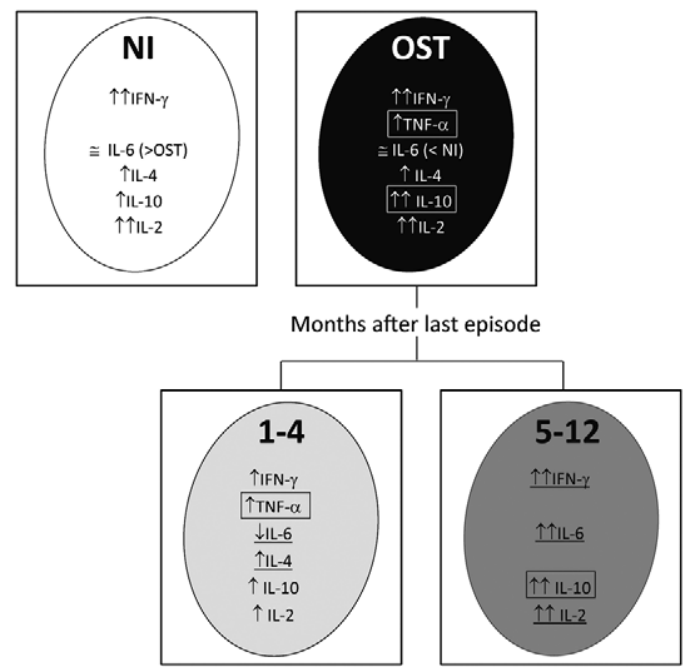

Fig. 4: schematic representation of two distinct cytokine signatures observed during early and late clinical episodes of chronic staphylococcal osteomyelitis. Increased frequency of high interferon (IFN)- $\gamma$, interleukin (IL)-10, IL-6 and IL-2-cytokine producers is observed at the late stage of chronic osteomyelitis, however the early stage is characterized by low frequency of individuals considered high producers of IL-6 associated with an increased frequency of high producers of tumour necrosis factor (TNF)- $\alpha$ and IL-4 after stimulation with staphylococcal enterotoxin A (SEA). NI: non-infected individuals; OST: osteomyelitis patients.

ticipate in the down-regulation during the late phase of chronic osteomyelitis. Additionally, IL-10 can inhibit the production of IFN- $\gamma$, IL-1 and TNF- $\alpha$ (Moore et al. 2001). IL-10 is also produced by different $\mathrm{T}$ lymphocyte sub-populations, including type I auxiliary T cells with regulatory activity (Jankovic \& Trinchieri 2007). These data suggest a role for IL-10 in modulating the late stage of chronic osteomyelitis. Therefore, the shift towards a regulatory immune response represents a dynamic event of pro-inflammatory and anti-inflammatory cytokine release in the transition from early to late chronic osteomyelitis.

In summary, the early stage of chronic osteomyelitis was characterised by increased levels of TNF- $\alpha$ and IL- 4 and reduced levels of IL-6 in SEA-stimulated PBMCs. The mixed TNF- $\alpha$ and IL-10 cytokine profile observed in chronic staphylococcal OST patients was the result of two distinct cytokine signatures that were observed during early and late clinical episodes. Finally, the overall cytokine signature triggered upon in vitro stimulation with SEA demonstrates that there is a distinct cytokine profile for IL-6 during early (1-4 months) and late (5-12 months) chronic osteomyelitic episodes.

\section{ACKNOWLEDGEMENTS}

To the members of the Laboratório de Biomarcadores de Diagnóstico e Monitoração, René Rachou Institute-Fiocruz/ MG, for providing invaluable technical assistance during this study, to PDTIS-Fiocruz, for use of the flow cytometry facili- ties, to our collaborators from the Hospital Regional de Governador Valadares, $\mathrm{MG}$, for their dedication to this study, and to Maria F da Silva, Marlucy R Lima, Lilia C Pires and Ivanete S Nascimento, for technical assistance.

\section{REFERENCES}

Abrahmsén L, Dohlsten M, Segrén S, Björk P, Jonsson E, Kalland T 1995. Characterization of two distinct MHC class II binding sites in the superantigen staphylococcal enterotoxin A. EMBO J 14: $2978-2986$.

Adwan GM, Abu-Shanab BA, Adwan KM, Jarrar NR 2006. Toxigenicity of Staphylococcus aureus isolates from northern Palestine. Emirates Med J 24: 1-3.

Asensi V, Montes AH, Valle E, Ocaña MG, Astudillo A, Alvarez V, Lópes-Anglada E, Solís A, Coto E, Meana A, Gonzales P, Carton JA, Paz J, Fieres J, Celada A 2007. The NO3 (27-bp repeat, intron 4) polymorphism is associated with susceptibility to osteomyelitis. Nitric Oxide 16: 44-53.

Asensi V, Valle E, Meana A, Fierer J, Celada A, Alvarez V, Paz J, Coto E, Carton JA, Maradona JA, Dieguez A, Sarasúa J, Ocaña MG, Arribas JM 2004. In vivo interelukin-6 protects neutrophils from apoptosis in osteomyelitis. Infect Immun 77: 3823-3828.

Balto K, Sasaki H, Stashenko P 2001. Interleukin-6 deficiency increases inflammatory bone destruction. Infect Immun 69: 744-750.

Bansal V, Mittal P, Ashokraj G 1992. Humoral immune responses in osteomyelitis. Int Orthop 16: 297-301.

Beharka AA, Iandolo JJ, Chapes SK 1995. Staphylococcal enterotoxins bind $\mathrm{H}-2 \mathrm{Db}$ molecules on macrophages. Proc Natl Acad Sci 92: 6294-6298.

Brady R, Leid J, Calhoun J, Costerton J, Shirtliff M 2008. Osteomyelitis and the role of biofilms in chronic infection. FEMS Iтmиnol Med Microbiol 52: 13-22.

Cardoso H, Silva N, Sena M, Carmo L 1999. Production of enterotoxins and toxic shock syndrome toxin by Staphylococcus aureus isolated from bovine mastitis in Brazil. Lett Appl Microbiol 29: 347-349.

Carek PJ, Dickerson LM, Sack JL 2001. Diagnosis and management of osteomyelitis. Am Fam Physician 63: 2413-2420.

Cho J, Cho S, Lee K 2009. Roles of SEA-expressing Staphylococcus aureus isolated from an atopic dermatitis patient on expressions of human beta-defensin-2 and inflammatory cytokines in $\mathrm{HaCaT}$ cells. Int J Mol Med 23: 331-335.

Dormans J, Drummond D 1994. Pediatric hematogenous osteomyelitis: new trends in presentation, diagnosis and treatment. $J \mathrm{Am}$ Acad Orthop Surg 2: 333-341.

Evans C, Jellis J, Hughes S, Remick D, Friedland J 1998. Tumor necrosis factor-a, interleukin- 6 and interleukin- 8 secretion and the acute-phase response in patients with bacterial and tuberculous osteomyelitis. J Infect Dis 177: 1582-1587.

Fullilove S, Jellis J, Hughes S, Remick D, Friedland J 2000. Local and systemic concentrations of tumor necrosis factor-a, interleukin- 6 and interleukin-8 in bacterial osteomyelitis. Trans $R$ Soc Trop Med Hyg 94: 221-224.

Gabay C 2006. Interleukin-6 and chronic inflammation. Arthritis Res Ther 8: 1-6.

Gazzinelli G, Katz N, Rocha R, Colley D 1983. Immune responses during human schistosomiasis mansoni. X. Production and standardization of an antigen-induced mitogenic activity by peripheral blood mononuclear cells from treated, but not active cases of schistosomiasis. J Immunol 130: 2891-2895. 
Gillespie WJ, Allardyce RA 1990. Mechanisms of bone degradation in infection: a review of current hypotheses. Orthopedics 13: 407-410.

Haas DW, McAndrew MP 1996. Bacterial osteomyelitis in adults: evolving considerations in diagnosis and treatment. $\mathrm{Am} \mathrm{J} \mathrm{Med}$ 101: $550-561$.

Harle A 1989. Infection management in total replacement. Arch Orthop Trauma Surg 108: 63-71.

Hierholzer S, Hierholzer G 1982. The unsuccessful surgical management of post-traumatic chronic bone infection. What is the role of serum factors? Arch Orthop Trauma Surg 100: 67-68.

Howell WR, Goulston C 2011. Osteomyelitis: an update for hospitalists. Hosp Pract (Minneap) 39: 153-160.

Hurst SM, Wilkinson TS, McLoughlin RM, Jones S, Horiuchi S, Yamamoto N, Rose-John S, Fuller GM, Topley N, Jones SA 2001. IL-6 and its soluble receptor orchestrate a temporal switch in the pattern of leukocyte recruitment seen during acute inflammation. Immunity 14: 705-714.

Jankovic D, Trinchieri G 2007. IL-10 or not IL-10: that is the question. Nature Immunol 8: 1281-1283.

Jevon M, Guo C, Ma B, Mordan N, Nair S, Harris M, Henderson B, Bentley G, Meghji S 1999. Mechanisms of internalization of Staphylococcus aureus by cultured human osteoblasts. Infect Immun 67: 2677-2681.

Jones SA, Richards PJ, Scheller J, Rose-John S 2005. IL-6 transsignalling: the in vivo consequences. J Interferon Cytokine Res 25: $241-253$

Jorge LS, Chueire AG, Rossit ARB 2010. Osteomyelitis: a current challenge. Braz J Infect Dis 14: 310-315.

Kaplanski G, Marin V, Montero-Julian F, Mantovani A, Farnarier C 2003. IL-6: a regulator of the transition from neutrophil to monocyte recruitment during inflammation. Trends Immunol 24: 25-29.

Klosterhalfen B, Peters KM, Tons C, Hauptmann S, Klein CL, Kirkpatrick CJ 1996. Local and systemic inflammatory mediator release in patients with acute and chronic posttraumatic osteomyelitis. J Trauma 40: 372-378.

Lew DP, Waldvogel FA 1997. Osteomyelitis. $N$ Engl J Med 336: 999-1007.

Lew DP, Waldvogel FA 2004. Osteomyelitis. Lancet 364: 369-379.

Marin V, Montero-Julian FA, Gres S, Boulay V, Bongrand P, Farnarier C, Kaplanski G 2001. The IL-6/soluble IL-6Ra autocrine loop of endothelial activation as an intermediate between acute and chronic inflammation: an experimental model involving thrombin. J Immunol 167: 3435-3442.

Marriot I, Gray DL, Tranguch SL, Fowler VG, Stryjewski M, Levin LS, Hudson MC, Bost KL 2004. Osteoblasts express the inflammatory cytokine interleukin-6 in a murine model of Staphylococcus aureus osteomyelitis and infected human bone tissue. Am J Path 164: 1399-1406.

Moore KM, Malefyt RW, Coffman RL, O'Garra A 2001. Interleukin-10 and the interleukin-10 receptor. Аnпи Rev Immunol 19: 683-765.
Moshage H, Jansen P 1998. Adaptation of nitrite and Griess method for measurement of serum nitrate plus nitrite levels. Ann Clin Biochem 35: 154-155.

Naffa GN, Bdour SM, Migdadi HM, Shehabi AA 2006. Enterotoxicity and genetic variation among clinical Staphylococcus aureus isolates in Jordan. J Med Microbiol 55: 183-187.

Nair SP, Willians RJ, Henderson B 2000. Advances in our understanding of the bone and joint pathology caused by Staphylococcus aureus infection. Rheumatology 39: 821-834.

Pesanti EL, Lorenzo JA 1998. Osteoclasts and effects of interleukin 4 in development of chronic osteomyelitis. Clin Orthop Relat Res 355: 290-299.

Peters KM, Koberg K, Kehren H, Zilkens KW 1991. PMN-elastase as a marker in diagnosis and follow-up of bone and joint infections. Unfallchirurg 94: 376-379.

Peters KM, Koberg K, Schmutzler W, Zwadlo-Klarwasser G 1995. Alteration in the pattern of macrophage subtypes in chronic osteomyelitis compared with acute joint infection. Int Orthop 19: 162-166.

Pudil R, Karpas R, Hanovcova I 1993. Basic indicators of cellular immunity in patients with chronic staphylococcal osteomyelitis. Acta Chir Orthop Traumatol Cech 60: 100-103.

Roesgen M, Hierholzer G, Hax PM 1989. Post-traumatic osteomyelitis. Pathophysiology and management. Arch Orthop Trauma Surg 108: 1-9.

Romano M, Sironi M, Toniatti C, Polentarutti N, Fruscella P, Ghezzi P, Faggioni R, Luini W, van Hinsbergh V, Sozzani S 1997. Role of IL- 6 and its soluble receptor in induction of chemokines and leukocyte recruitment. Immunity 6: 315-325.

Sasaki S, Miura T, Nishikawa S, Yamada K, Hirasue M, Nakane A 1998. Protective role of nitric oxide in Staphylococcus aureus infection in mice. Infect Immun 66: 1017-1022.

Schluter B, Bergmann U, Josten C, Walz M, Konig W 1991. Impairment of specific host defense mechanisms in patients with chronic post-traumatic osteomyelitis. $J$ Trauma 31: 68-73.

Sistermann R, Möllenhoff G, Walz M, Josten C, Muhr G 1992. A cellular quantitative immune deficiency in chronic post-traumatic osteomyelitis. Unfallchirurg 95: 254-258.

Sourek J, Vmola F, Trojanová M, Zelenková L, Matjovska V, Bergdoll MS 1979. Enterotoxin production by Staphylococcus aureus strains isolated from cases of chronic osteomyelitis. J Clin Microbiol 9: 266-268.

Tsezou A, Poultside L, Kostopoulou F, Zintzaras E, Satra M, KitsiouTzeli S, Malizos KN 2008. Influence of interleukin 1 (IL-1), IL-4 and IL-6 polymorphisms on genetic susceptibility to chronic osteomyelitis. Clin Vaccine Immunol 15: 1888-1890.

Wagner C, Kondella K, Bernschneider T, Heppert V, Wentzensen A, Hansch M 2003. Post-traumatic osteomyelitis: analysis of inflammatory cells recruited into the site of infection. Shock 20: 503-510.

Yoon K, Fitzgerald-Jr R, Sud S, Song Z, Wooley P 1999. Experimental acute haematogenous osteomyelitis in mice. II. Influence of Staphylococcus aureus infection on T-cell immunity. J Orthop Res 17: 382-391. 